\title{
Time to Death and Its Predictors Among Neonates Admitted With Neonatal Sepsis at Public Referral Hospitals of Bahir Dar City, Northwest Ethiopia, 2021: Retrospective Cohort Study
}

Mekdes Tadesse Abebia ( $\sim$ mekdestadesse88@yahoo.com ) Bahir Dar University College of Medical and Health Sciences https://orcid.org/0000-0003-0016-2889

Getasew Tesfa Kibret

Bahir Dar University College of Medical and Health Sciences

Minyichil Birhanu Belete

Bahir Dar University College of Medical and Health Sciences

Azeb Geddif Asmare

Bahir Dar University College of Medical and Health Sciences

Mahlet Tamirat Abate

Bahir Dar University College of Medical and Health Sciences

Silenat Muluken Abebe

Bahir Dar University College of Medical and Health Sciences

Haileyesus Gedamu Wondfraw

Bahir Dar University College of Medical and Health Sciences

Dagmawit Zewdu Wuletaw

Bahir Dar University College of Medical and Health Sciences

Research

Keywords: neonatal period, neonatal sepsis, time to death, predictors

Posted Date: October 25th, 2021

DOl: https://doi.org/10.21203/rs.3.rs-990326/v1

License: (c) (1) This work is licensed under a Creative Commons Attribution 4.0 International License.

Read Full License 


\section{Abstract}

Background: In spite of recent advances in health care, neonatal sepsis is still one of the major causes of morbidity and mortality in neonates and is an ongoing major global public health challenge. Its incidence of mortality varies from health institution to health institution and within the same health institution at varied times and depends on predisposing factors. So the aim of this study was to determine the time to death and its predictors among neonates admitted with neonatal sepsis.

Methods: An institutional based retrospective cohort study was conducted among 500 randomly selected neonatal charts. The data was entered using Epi data version 3.1, and exported to and analyzed at STATA version 14. Bivariable and multivariable cox regression analysis were conducted to identify predictors of mortality. Association was summarized using adjusted hazard ratio (AHR) and statistical significances were declared at $95 \% \mathrm{Cl}$ and P-value $<0.05$. Proportionality assumption was tested by global test based on Schoenfeld residuals analysis.

Results: During the follow up time, $58(11.6 \%)$ neonates were died with neonatal sepsis while the rest were censored. In this study the overall death incidence rate was 20.5 per 1000 neonate days.

Comorbidity (AHR: 1.81, 95\%, Cl: 1.04, 3.17), late initiation of exclusive breast feeding over one hour (AHR: $2.29,95 \% \mathrm{Cl}: 1.13,4.63)$, history of intra-partum fever(AHR: $7.3795 \% \mathrm{Cl}: 2.28,23.79)$, Birth weight (AHR: 3.37, 95\%Cl: $1.54,7.34$ ) and place of delivery(AHR: $3.83,95 \%$, Cl: $1.24,11.83$ ) were found potential independent predictors of mortality among neonates with neonatal sepsis.

Conclusion: In this study, high death incidence rate was observed. The mean time to death among neonates was 4.41 days. With regard to predictors: birth weight, comorbidity, late initiation of exclusive breast feeding over one hour, history of intra partum fever and place of delivery were significant predictors of mortality among neonates admitted with neonatal sepsis.

\section{Background}

Neonatal sepsis is a clinical syndrome in an infant 28 days of life or younger, manifested by systemic signs of infection and isolation of a bacterial pathogen from the bloodstream $(1,2)$. According to the international pediatric sepsis consensus conference of 2005, neonatal sepsis can also be defined as systemic inflammatory response syndrome in the presence of or as a result of suspected or proven infection in a neonate which presents with clinical features of temperature instability, respiratory problems, feeding intolerance, and isolation of bacteria or other pathogens from the bloodstream(3). Neonatal sepsis occurs when pathogenic bacteria gain access into the blood stream causing overwhelming general infection. The most common isolated bacteria were gram-negative(4).

Neonatal sepsis is classified into two major categories based on the time of onset: Early-onset neonatal sepsis (EONS) and Late-onset neonatal sepsis (LONS). Early onset neonatal sepsis appears within the first seven days of life and is mainly due to bacteria acquired before and during delivery and most cases appear with in the first 24 hours(5). According to the World Health Organization (WHO) report in 2006, out 
of the 130 million live births every year, 4 million die within the first four weeks of life. Of these deaths, $99 \%$ occur in developing countries (6). Neonatal sepsis is accountable for $15 \%$ of global neonatal deaths and $30-50 \%$ of neonatal deaths in developing countries (7). In sub Saharan Africa, $17 \%$ of neonatal deaths are due to neonatal sepsis (8). Around $37 \%$ of Ethiopian neonates also died due to neonatal sepsis, which accounts for more than one third of neonatal deaths (9).

Sepsis is still one of the major causes of morbidity and mortality in neonates. In spite of recent advances in health care units, it is an ongoing major global public health challenge leaving the survivors vulnerable to short and long-term neurodevelopmental morbidity (10). Among those who survive, impaired quality of life, increased dependency, re-hospitalization, increase healthcare consumption, and along with increased mortality, all contribute the humanistic and economic burden of sepsis(11). Neonates could also face long-term survival with post infectious hydrocephalus (PIH), which could result in childhood mortality or survival with $\mathrm{PIH}$ with resulting neurological impairment and this long-term survival from sepsis with neurodevelopmental impairment could result in cerebral palsy or lifetime neurodevelopmental impairment without cerebral palsy; or uncomplicated recovery following an acute 2-week period of neonatal sepsis (12).

Different studies have been conducted in developed and developing countries to determine incidence density rate of neonatal mortality with sepsis. A study done in Brazil showed that the incidence and the incidence density of sepsis was $22 \%$ and 18.6 per 1000 central venous catheter-days, respectively. Considering very low birth weight neonates (VLBW) neonates as the reference group, the hazard ratio (HR) for time to death was 4.06 from day 0 to 60 and for time to the first episode of sepsis was 1.76 from day 0 to 36 . Having the heavier neonates group as reference, the HR for time to the first episode of sepsis was 2.94 from day 37 to 90 (13).

A study conducted in Tanzania revealed a mortality rate of $19 \%$ in neonates with sepsis and high mortality is seen in early onset neonatal sepsis and showed a survival rate of $47 \%$ (14). Another study conducted in Uganda shows that mortality rate due to neonatal sepsis is $18.1 \%$ and most of the deaths (84\%) occurred in the first 48 hours of admission(15). In Nigeria a study indicate that mortality rate due to sepsis was $15.7 \%$. In this study, low birth weight was 7.2 times more likely to die compared to those who were appropriate for birth weight. Babies that had PROM were 2.5 times more likely to die(16). Another study done in Johannesburg, showed the overall mortality rate for neonatal sepsis was $1.7 \%(17)$.

A study done in Sub-Saharan Africa to estimate the incidence density rate of neonatal mortality among neonates the incidence density rate of mortality was significantly high. And the hazard of neonatal mortality in NICUs was increased by comorbidities like sepsis with the hazard of death of neonates with sepsis was 1.94 times than neonates free from sepsis (18).

A study in southern Ethiopia revealed that the incidence rate of mortality among neonates admitted with neonatal sepsis was 14.57 per 1000 neonate days. The cumulative proportion of surviving at the end of the fourth day was $99.5 \%$, and it was $98.2 \%$ at the end of the fifth day. In addition, it was $96.6 \%, 93.5 \%$, and $91.1 \%$ at the end of the sixth, seventh, and eighth day, respectively (1). 
Prolonged rupture of membrane(>48hrs), maternal fever, and chorioamnionitis were found significant predictors of mortality of neonates with sepsis in a retrospective study conducted in Pakistan (19).

Efforts to reduce hospital acquired late onset infections require much closer attention to appropriate hand washing, infection control, and proper techniques for placement and management of central catheters (20). Antenatal corticosteroid treatment for accelerating fetal lung maturation for women at risk of preterm birth has been shown to reduce birth complications including the risk of neonatal sepsis (21).

Even though the impact of neonatal sepsis remains a public health problem in resource limited settings like Ethiopia, limited evidences exist to show the time to death and predictors of this serious public health problem in different parts of Ethiopia particularly in the study setting. Thus, determining the time to death and identifying its predictors among neonates with neonatal sepsis is very important for optimizing neonatal care.

Though Ethiopia has made considerable achievement in the reduction of under-five mortality rate, the neonatal mortality burden has not experienced the same reduction, which may be attributed to neonatal sepsis. Neonatal sepsis is a relevant public health issue because it consistently emerges as one of the main causes of neonatal morbidity and mortality. Therefore, evidence on time to death of neonates with sepsis and factors that predicts the death of neonates with sepsis in the area will provide knowledge for health professionals in early identification of high-risk neonates with sepsis and timely intervention for their better survival. It also enhances the current neonatal sepsis management strategies by making sure the various determinants contribute to neonatal sepsis. This study will also be used for future researchers as reference. Also identifying the predictors of neonatal sepsis will be helpful in designing strategies to prevent and/or treat neonatal sepsis. Therefore, the aim of this study is to determine time to death and identifying predicting factors for death among neonates admitted with sepsis at public hospitals of Bahir Dar city.

\section{Methods}

\section{Study design, period, and settings}

An institutional- based retrospective cohort study design was carried out from February 1, 2021 to March 1, 2021 among neonates with neonatal sepsis at Bahir Dar city public referral hospitals admitted from January 1, 2019 to December 31, 2020. This study was conducted in Bahir Dar city public referral hospitals namely: Felege Hiwot comprehensive specialized hospital and Tibebe Ghion specialized hospital. Bahir Dar city is the capital city of Amhara regional state located in the Northwest Ethiopia which is found $565 \mathrm{~km}$ away from Addis Ababa, the capital city of Ethiopia.

\section{Study participants, sample size and sampling technique}

All neonates admitted with neonatal sepsis were the study population and those neonates age less than or equal to 28 days at admission and were admitted during January 1, 2019 to December 31, 2020 were 
included in the study. On the contrary neonates with incomplete chart records were excluded from the study. The minimum sample size required was determined with Epi info version 7 statistical packages using major predictor variables from previous study (1), by considering the following statistical assumptions: $\mathrm{Cl}=95 \%$, and power $=80 \%$. Accordingly, onset of labor was an independent variable which gives maximum sample size of $\mathbf{4 5 4}$ as mentioned in the table below. Then by adding $10 \%$ contingency, the final sample size was $\mathbf{5 0 0}$

\section{Sampling procedure}

First, medical registration numbers of all neonates with neonatal sepsis admitted for the last two years (January 1, 2019 to December 31, 2020) were obtained from the hospitals' registration logbooks. Then samples were selected by simple random sampling technique using computer generating system, after having lists of the medical registration number of the neonatal charts with neonatal sepsis from both hospitals.

\section{Data extraction procedures}

A data extraction tool was adapted from the Ethiopian federal ministry of health, national standardized neonatal and delivery registration book and prepared in English as structured checklist to extract required data from selected charts. The checklist include; socio demographic factors of both maternal and neonatal, neonatal factors and maternal factors. The data was collected from the $1 \mathrm{st}$ date of admission to death or censored (lost to follow up, improved, referred or against medical treatment) or until the end of follow up period ( 28 days of age).The data was extracted with four BSc. nurses who had work experience in NICU and two MSc. nurses were also recruited for supervision throughout the data collection period. Training was given for data collectors and supervisors two days before data collection. Pretest was done in $5 \%$ of neonatal charts with neonatal sepsis in FHCSH 5 days prior to data collection period and inconsistencies were checked and corrected. A close supervision was carried out by the supervisors during data collection period. Finally, all the collected data was checked by investigator for its completeness and consistency.

\section{Operational definitions}

Neonate: A new born from birth until 28 days old

Neonatal sepsis: Sepsis diagnosed and confirmed by physician among neonates.

Early-onset neonatal sepsis: Sepsis diagnosed and confirmed by physician among neonates age less than seven days.

Late-onset neonatal sepsis: Sepsis diagnosed and confirmed by physician among neonates age seven days or more.

Event: Death of neonate admitted with neonatal sepsis. 
Censored: Neonates with other than event/death (Improved and discharged, referred to other health facility, against medical treatment)

Time to death: The time in days from admission to death of neonates with neonatal sepsis

Follow up time: Neonatal age period (neonate with neonatal sepsis from admission until either event or censorship occurs).

\section{Data processing and analysis}

Data was cleaned, edited and coded by using Epi data version 3.1. Consistency of data was also checked before analysis to avoid errors during data entry. After that, it was exported to STATA 14 statistical software. Descriptive statistics (mean, standard deviation, frequency and percentages) were computed depending on the nature of variable. Outcome of each participant was dichotomized into censored or death. Incidence density rate (IDR) was calculated for the entire study period. Failure probability table and Kaplan-Meir (KM) failure function was used to estimate the mean time to death and cumulative probability of failure. KM plot and log-rank tests were used to compare failure curves. Before performing The Cox-proportional hazard regression, model, goodness-of-fit was checked by Cox Snell residuals and assumptions were also checked by using Schoenfeld residual test. For each independent predictor bivariable cox proportional Hazard regression was performed. Then the variables with p-vale $<0.2$ were included in multivariable cox proportional hazard regression. AHR with $95 \%$ confidence interval and $p$ values $<0.05$ was used to measure the strength of association and identify statistical significant predictors.

\section{Results}

\section{Socio-demographic characteristics of the mothers and neonates}

In this study, 500 neonatal charts were reviewed. More than half 296(59.2\%) were males which accounted for $70.69 \%$ of dead neonates as compared with female neonates. The mean ( \pm SD) age of the neonates was $4.41 \pm 6.75$ days. About $82.6 \%$ of the neonates had age less than seven days. Among the neonates who died with neonatal sepsis, $37(77.08 \%)$ of them died within the first seven days of life. Concerning to mothers' socio demographic characteristics, the mean $( \pm S D)$ age of mothers was $27.44 \pm 5.32$ years range from 15 to 44 years (Table 1 ). 
Table 1

Socio-demographic characteristics of neonates admitted with neonatal sepsis at public referral hospitals of Bahir Dar city, northwest, Ethiopia, 2021 ( $n=500)$

\begin{tabular}{|c|c|c|c|}
\hline \multirow[t]{2}{*}{ Variables } & \multirow[t]{2}{*}{ Category } & \multicolumn{2}{|c|}{ Status of the neonate } \\
\hline & & Death & Censored \\
\hline \multirow[t]{3}{*}{ Maternal age } & $<20$ & $1(1.72 \%)$ & $15(3.39 \%)$ \\
\hline & $20-34$ & $45(77.59 \%)$ & $358(81 \%)$ \\
\hline & $>34$ & $12(20.69 \%)$ & $69(15.61 \%)$ \\
\hline \multirow[t]{2}{*}{ Neonatal age } & $<7$ & $47(81.03 \%)$ & $366(82.81 \%)$ \\
\hline & $7-28$ & 11(18.97\%) & 76(17.19\%) \\
\hline \multirow[t]{2}{*}{ Neonatal sex } & Male & $41(70.69 \%)$ & $255(57.69 \%)$ \\
\hline & Female & 17(29.31\%) & $187(42.31 \%)$ \\
\hline
\end{tabular}

\section{Obstetrics-related characteristics}

In this study nearly three fourth $357(71.4 \%)$ of the mothers were primigravida and majority 38 (65.52\%) death was observed in this category. This study result also showed that most of the mothers $483(96.6 \%)$ have received ANC service during their pregnancy and similarly almost all 55 (94.83\%) deaths of neonates were observed among mothers having ANC follow up. With regard to onset of labor, majority $448(89.6 \%)$ had spontaneous onset of labor and again the majority of the mothers $485(97 \%)$ delivered their newborn at health institution and more than half 344 (68.8\%) of them delivered by spontaneous vaginal delivery. Regarding rupture of membrane, 122 (24.4\%) had history of PROM and out of them 49 $(40.16 \%)$ were for more than 18 hour duration (Table 3$)$. 
Table 2

Obstetrics-related characteristics of mothers having neonates admitted with neonatal sepsis at public referral hospitals of Bahir Dar city, northwest, Ethiopia, 2021 ( $n=500)$

\begin{tabular}{|c|c|c|c|}
\hline \multirow[t]{2}{*}{ Variables } & \multirow[t]{2}{*}{ Category } & \multicolumn{2}{|c|}{ Status of the neonate } \\
\hline & & Death & Censored \\
\hline \multirow[t]{3}{*}{ Gravidity } & Primi gravida & $38(65.52 \%)$ & $319(72.17 \%)$ \\
\hline & Mult gravida & $11(18.97 \%)$ & $79(17.87 \%)$ \\
\hline & Grandmulti gravida & $9(15.52 \%)$ & $44(9.95 \%)$ \\
\hline \multirow[t]{3}{*}{ Parity } & Primi para & $39(67.24 \%)$ & $325(73.53 \%)$ \\
\hline & Multi para & $11(18.97 \%)$ & 79 (17.87 \%) \\
\hline & Grandmulti para & $8(13.79 \%)$ & $38(8.6 \%)$ \\
\hline \multirow[t]{2}{*}{ ANC follow up } & Yes & $55(94.83 \%)$ & $428(96.83 \%)$ \\
\hline & No & $3(5.17 \%)$ & $14(3.17 \%)$ \\
\hline \multirow[t]{2}{*}{ Number of ANC visit } & $1-3$ & $25(45.45 \%)$ & $97(22.66 \%)$ \\
\hline & $\geq 4$ & $30(54.55 \%)$ & $331(77.34 \%)$ \\
\hline \multirow[t]{2}{*}{ Onset of labor } & Spontaneous & $54(93.1 \%)$ & $394(89.14 \%)$ \\
\hline & Induced & $4(6.9 \%)$ & $48(10.86 \%)$ \\
\hline \multirow[t]{2}{*}{ Place of delivery } & Health institution & $54(93.1 \%)$ & $431(97.51 \%)$ \\
\hline & Home & $4(6.9 \%)$ & $11(2.49 \%)$ \\
\hline \multirow[t]{5}{*}{ Mode of delivery } & Spontaneous vaginal & $43(74.14 \%)$ & $301(68.1 \%)$ \\
\hline & Delivery & & \\
\hline & Assisted instrumental & $7(12.01 \%)$ & $30(6.79 \%)$ \\
\hline & Delivery & & \\
\hline & Cesarean section & $8(13.79 \%)$ & $111(25.11 \%)$ \\
\hline \multirow[t]{2}{*}{ History of PROM } & Yes & $18(31.03 \%)$ & $104(23.53 \%)$ \\
\hline & No & $40(68.97 \%)$ & $338(76.47 \%)$ \\
\hline \multirow[t]{2}{*}{ Duration of PROM } & $<18$ hours & $10(55.56 \%)$ & $63(60.58 \%)$ \\
\hline & $\geq 18$ hours & $8(44.44 \%)$ & $41(39.42 \%)$ \\
\hline \multirow[t]{2}{*}{ History of foul smelling liquor } & Yes & $5(8.62 \%)$ & $14(3.17 \%)$ \\
\hline & No & $53(91.38 \%)$ & $428(96.83 \%)$ \\
\hline
\end{tabular}




\begin{tabular}{|llll|}
\hline Variables & Category & \multicolumn{2}{l|}{ Status of the neonate } \\
\hline History of fever & Yes & $4(6.9 \%)$ & $3(0.68 \%)$ \\
\cline { 2 - 4 } & No & $54(93.1 \%)$ & $439(99.32 \%)$ \\
History of preeclampsia & Yes & $3(5.17 \%)$ & $21(4.75 \%)$ \\
\cline { 2 - 4 } History of APH & No & $55(94.83 \%)$ & $421(95.25 \%)$ \\
History of chorioamniotis & Yes & $2(3.45 \%)$ & $3(0.68 \%)$ \\
& No & $56(96.55 \%)$ & $439(99.32 \%)$ \\
\cline { 2 - 4 } & No & $3(5.17 \%)$ & $29(6.56 \%)$ \\
\hline
\end{tabular}

\section{Neonatal related characteristics of neonates with neonatal sepsis}

From 500 neonates admitted with neonatal sepsis, the mean ( \pm SD) weight of neonates at admission was $2715.4 \pm 643.8$ grams and more than half, $37(63.79 \%)$ of death was observed among neonates with admission age less than 2500 grams. Regarding gestational age, the mean ( \pm SD) gestational age was $38.1 \pm 2.7$ weeks ranging from 29-45 weeks. Among dead neonates majority, 49 (84.48) of them were neonates who had APGAR score less than seven at the first minute and $37(63.79 \%)$ of dead neonates had APGAR score less than seven at fifth minute (Table 3). 
Table 3

Neonatal related characteristics of neonates admitted with neonatal sepsis at public referral hospitals of Bahir Dar city, northwest, Ethiopia, 2021 ( $n=500)$

\begin{tabular}{|c|c|c|c|}
\hline \multirow[t]{2}{*}{ Variables } & \multirow[t]{2}{*}{ Category } & \multicolumn{2}{|c|}{ Status of the neonate } \\
\hline & & Death & Censored \\
\hline \multirow[t]{2}{*}{ Gestational age } & Preterm & $30(51.72 \%)$ & $97(21.95 \%)$ \\
\hline & Term & $28(48.28 \%)$ & $345(78.05 \%)$ \\
\hline \multirow[t]{2}{*}{ Admission weight } & $<2500$ & $37(63.79 \%)$ & $86(19.46 \%)$ \\
\hline & $\geq 2500$ & $21(36.21 \%)$ & $356(80.54 \%)$ \\
\hline \multirow[t]{2}{*}{ Comorbidity } & Yes & $31(53.45 \%)$ & $133(30.09 \%)$ \\
\hline & No & $27(46.55 \%)$ & $309(69.91 \%)$ \\
\hline \multirow[t]{2}{*}{ First minute APGAR score } & $<7$ & 49 (84.48 \%) & $276(62.44 \%)$ \\
\hline & $\geq 7$ & $9(15.52 \%)$ & $166(37.56 \%)$ \\
\hline \multirow[t]{2}{*}{ Fifth minute APGAR score } & $<7$ & $37(63.79 \%)$ & $112(25.34 \%)$ \\
\hline & $\geq 7$ & $21(36.21)$ & $330(74.66 \%)$ \\
\hline \multirow[t]{2}{*}{ Cry immediately at birth } & Yes & $23(39.66 \%)$ & $372(82.96 \%)$ \\
\hline & No & $35(60.34 \%)$ & $70(15.84 \%)$ \\
\hline \multirow[t]{2}{*}{ Resuscitated at birth } & Yes & $20(34.48 \%)$ & $41(9.28 \%)$ \\
\hline & No & $38(65.52 \%)$ & $401(90.72 \%)$ \\
\hline \multirow[t]{2}{*}{ EBF initiated within one hour } & Yes & $18(31.03 \%)$ & $297(67.19 \%)$ \\
\hline & No & $40(68.97 \%)$ & $145(32.81 \%)$ \\
\hline \multirow[t]{2}{*}{ History of previous hospitalization } & Yes & $12(20.69 \%)$ & $53(11.99 \%)$ \\
\hline & No & $46(79.31 \%)$ & $389(88.01 \%)$ \\
\hline
\end{tabular}

\section{Outcome of neonates with neonatal sepsis}

During follow up time, 58(11.6\%) neonates with neonatal sepsis were died while 442(88.4\%) of neonates with neonatal sepsis were censored of which, $(77 \%$ of the neonates were improved and discharged, $8.2 \%$ of the neonates were against medical treatment and $3.2 \%$ of the neonates were referred to other hospitals) (figure 1).

\section{Failure/Death of Neonates}


The neonates were followed for a total of 2848 days. Among them, 58(11.6\% [95\% Cl: 9.07, 14.73]) died during the follow up time and that makes the mortality rate from neonatal sepsis 116 per 1000 live births. The overall death incidence rate in this study was 20.5 per 1000 neonate days ( $95 \% \mathrm{Cl}: 0.01,0.26)$.

From all deaths, $13 \%$ of the neonates were died in the first 24 hours of life, $20 \%$ of the neonates died in the first three days of life and about $81 \%$ of death occurred with in the first seven days of life. Death from neonatal sepsis was also further analyzed from the time of admission and consequently, $10.4 \%$ of the deaths occurred in the first 24 hours, $46.5 \%$ of the deaths occurred with in the first three days and about $90 \%$ of the deaths occurred within the first week of admission. At the end of follow up time, the cumulative failure probability was $28.13 \%$ (95\% Cl: 20.0438 .62$)$.

In this study since the maximum observation time was censored the median time to death was not determined therefore, mean time to death was the appropriate measure of central tendency. Accordingly, the mean time to death among neonates died was 4.41 days (95\% Cl: $3.62,5.21)$.

The probability of death in neonates with neonatal sepsis during the follow-up time was also presented by the failure curve. Accordingly, during the first seven days, the graph went up increasingly showing a higher probability of death. Also, between days 7 and 13, the probability of death continued and the graph moved upward. In the remaining days of the follow-up period, the graph became straight indicating the likelihood of death in neonates with neonatal sepsis remained stable with almost no deaths occurring (figure 2).

\section{Log-rank estimate of mortality among neonates with neonatal sepsis across predictors}

As shown in the table below the log-rank test estimate between categories of different predictor variables revealed that the survival pattern among neonates with neonatal sepsis was significantly different as there is a highly significant difference among survival curves. The Kaplan-meier together with the log-rank test shows the effect of each predictor on the neonatal mortality with neonatal sepsis (Table 4). 
Table 4

Log-rank test for equality of different categorical predictors of neonates admitted with neonatal sepsis at public referral hospitals, Bahir Dar city, northwest, Ethiopia, 2021 ( $n=500)$

\begin{tabular}{|c|c|c|c|}
\hline Variables & Category & $x^{2}$ & P-value \\
\hline \multirow[t]{2}{*}{ History of liquor } & Yes & 4.41 & 0.03 \\
\hline & No & & \\
\hline \multirow[t]{2}{*}{ First minute APGAR score } & $<7$ & 11.17 & $<0.001$ \\
\hline & $\geq 7$ & & \\
\hline \multirow[t]{2}{*}{ Fifth minute APGAR score } & $<7$ & 34.87 & $<0.001$ \\
\hline & $\geq 7$ & & \\
\hline \multirow[t]{2}{*}{ Admission weight } & $<2500$ & 42.97 & $<0.001$ \\
\hline & $\geq 2500$ & & \\
\hline \multirow[t]{2}{*}{ Cry immediately at birth } & Yes & 50.29 & $<0.001$ \\
\hline & No & & \\
\hline \multirow[t]{2}{*}{ Resuscitated at birth } & Yes & 20.44 & $<0.001$ \\
\hline & No & & \\
\hline \multirow[t]{2}{*}{ Gestational age } & Preterm & 24 & $<0.001$ \\
\hline & term & & \\
\hline \multirow[t]{2}{*}{ Place of delivery } & Health institution & 4.12 & 0.04 \\
\hline & Home & & \\
\hline \multirow[t]{2}{*}{ History of fever } & Yes & 58 & $<0.001$ \\
\hline & No & & \\
\hline \multirow[t]{2}{*}{ Comorbidity } & Yes & 12.59 & $<0.001$ \\
\hline & No & & \\
\hline \multirow[t]{2}{*}{ EBF initiated within one hour } & Yes & 33.93 & $<0.001$ \\
\hline & No & & \\
\hline
\end{tabular}

\section{Testing overall fitness of the model}

Model goodness of fit was checked by using the Cox-Snell residual test. The residuals had a standard censored exponential distribution with hazard ratio. The jagged line with the reference line (Cox Snell 
residual line) follows the $45^{\circ}$ line closely. Hence, the overall cox-regression model fits to the data (Figure 3).

\section{Cox proportional hazard assumption test}

For each covariate, Cox proportional hazard assumption was performed individually and simultaneously (Globally).The test showed that the p-value for each covariate and the whole covariates simultaneously were greater than 0.05 which showed that there were no time-varying covariates in the model (Table 5). 
Table 5

Test of cox proportional hazard assumption among neonates admitted with neonatal sepsis at public referral hospitals, Bahir Dar city, northwest, Ethiopia, 2021 $(n=500)$

\begin{tabular}{|c|c|c|c|c|}
\hline Variables & Rho & quare & $\mathrm{df}$ & Prob>chi2 \\
\hline Weight at admission & 0.00883 & 0.01 & 1 & 0.9371 \\
\hline Onset of labor & -0.07212 & 0.32 & 1 & 0.5700 \\
\hline Maternal age & -0.14465 & 1.38 & 1 & 0.2407 \\
\hline Gestational age & 0.15583 & 1.68 & 1 & 0.1946 \\
\hline History of previous hospitalizatio & on -0.08212 & 0.34 & 1 & 0.5576 \\
\hline EBF initiated within one hour & 0.07645 & 0.41 & 1 & 0.5218 \\
\hline Resuscitated at birth & -0.03881 & 0.13 & 1 & 0.7205 \\
\hline Cry immediately at birth & 0.13725 & 1.24 & 1 & 0.2655 \\
\hline Fifth minute APGAR score & -0.04866 & 0.18 & 1 & 0.6678 \\
\hline First minue APGAR score & 0.19293 & 2.17 & 1 & 0.1403 \\
\hline Medical problem in addition seps & sis 0.18874 & 2.36 & 1 & 0.1244 \\
\hline History of chorioamnionitis & 0.10922 & 0.63 & 1 & 0.4262 \\
\hline History of APH & 0.11192 & 1.04 & 1 & 0.3085 \\
\hline Preeclampsia & -0.14811 & 1.23 & 1 & 0.2677 \\
\hline History of fever & -0.19153 & 2.25 & 1 & 0.1338 \\
\hline History of liquor & -0.17774 & 1.66 & 1 & 0.1981 \\
\hline Mode of delivery & 0.18759 & 2.27 & 1 & 0.1316 \\
\hline History of PROM & 0.13339 & 1.03 & 1 & 0.3098 \\
\hline ANC follow up & -0.02266 & 0.02 & 1 & 0.8873 \\
\hline Number of Parity & 0.15200 & 1.41 & 1 & 0.2346 \\
\hline Gravidity & -0.07892 & 0.42 & 1 & 0.5177 \\
\hline Neonatal sex & -0.07437 & 0.35 & 1 & 0.5533 \\
\hline Neonatal age & -0.04940 & 0.17 & 1 & 0.6798 \\
\hline Global test & & 23.11 & 23 & 0.4545 \\
\hline
\end{tabular}




\section{Bivariable and multivariable cox proportional hazard regression model for different predictor variables}

Cox proportional hazard regression model was fitted and used to identify predictors of mortality in neonates with neonatal sepsis. In bivariate Cox proportional hazard regression: neonatal sex, gestational age, weight at admission, history of PROM, place of delivery, history of liquor, history of fever, comorbidity, cry immediately at birth, resuscitated at birth, EBF initiated within one hour, history of previous hospitalization, first minute APGAR score and fifth minute APGAR score were significant predictors of mortality among neonates with neonatal sepsis with $p$-value $<0.2$. In a multivariable proportional hazard model: admission weight, comorbidity, late initiation of EBF over one hour, history of fever and place of delivery remains potential independent predictors of mortality among neonates with neonatal sepsis at ( $p$ value<0.05).

Multivariable analysis resulted that neonates delivered at home had a 3.83 times higher hazard of death than neonates delivered at health institution (AHR: 3.83, 95\%, Cl: 1.24, 11.83). The hazard of death in neonates with admission weight <2500 grams was 3.37 times higher compared to neonates with admission weight $\geq 2500$ (AHR: $3.37,95 \% \mathrm{Cl}$ : 1.54, 7.34). Neonates who had comorbidity showed a 1.81 times higher hazard of death when compared to neonates who had no comorbidity (AHR: $1.81,95 \%, \mathrm{Cl}$ : $1.04,3.17)$. Neonates who were born from mothers who had a history of fever were 7.37 times more likely to die as compared to those neonates born from mothers with no history of fever (AHR: 7.37 95\%Cl:

$2.28,23.79$ ). Neonates who did not get initiated to exclusive breast feeding within one hour showed a 2.44 times higher hazard of death than neonates who did get initiated to exclusive breast feeding within one hour(AHR: 2.29, 95\%Cl: 1.13,4.63) (Table 6). 
Table 6

Bivariate and multivariate Cox proportional hazard regression outputs of neonates admitted with neonatal sepsis at public referral hospitals, Bahir Dar city, northwest, Ethiopia, $2021(n=500)$

\begin{tabular}{|c|c|c|c|}
\hline Covariates & Category & $\mathrm{CHR}(95 \% \mathrm{Cl})$ & $\operatorname{AHR}(95 \% \mathrm{Cl})$ \\
\hline \multirow[t]{2}{*}{ Neonatal sex } & Male & $1.58(0.90,2.78)$ & $1.82(1.00,3.31)$ \\
\hline & Female & 1 & 1 \\
\hline \multirow[t]{2}{*}{ Gestational age } & Preterm & $3.22(1.98,5.57)$ & $0.87(0.41,1.81)$ \\
\hline & Term & 1 & 1 \\
\hline \multirow[t]{2}{*}{ Weight at admission(gram) } & $<2500$ & $5.03(2.93,8.64)$ & $3.37(1.54,7.34)^{\star \star \star}$ \\
\hline & $\geq 2500$ & 1 & \\
\hline \multirow[t]{2}{*}{ Place of delivery } & Health institution & 1 & 1 \\
\hline & Home & $2.71(0.98,7.52)$ & $3.83(1.24,11.83)^{\star \star}$ \\
\hline \multirow[t]{2}{*}{ History of PROM } & Yes & $1.46(0.84,2.56)$ & $1.84(0.97,3.48)$ \\
\hline & No & 1 & 1 \\
\hline \multirow[t]{2}{*}{ History of Fever } & Yes & $6.15(2.21,17.16)$ & $7.37(2 \cdot 28,23 \cdot 79)^{\star \star \star}$ \\
\hline & No & 1 & 1 \\
\hline \multirow[t]{2}{*}{ History of liquor } & Yes & $2.55(1.02,6.41)$ & $0.76(0.26,2.29)$ \\
\hline & No & 1 & 1 \\
\hline \multirow[t]{2}{*}{ Comorbidity } & Yes & $2.45(1.46,4.10)$ & $1.81(1.04,3.17)^{\star \star}$ \\
\hline & No & 1 & 1 \\
\hline \multirow[t]{2}{*}{ First minute APGAR score } & $<7$ & $3.13(1.53,6.34)$ & $1.33(0.54,3.29)$ \\
\hline & $\geq 7$ & 1 & 1 \\
\hline \multirow[t]{2}{*}{ Fifth minute APGAR score } & $<7$ & $4.31(2.52,7.36)$ & $1.14(0.53,2.45)$ \\
\hline & $\geq 7$ & 1 & 1 \\
\hline \multirow[t]{2}{*}{ Cry immediately at birth } & Yes & 1 & 1 \\
\hline & No & $5.44(3.18,9.16)$ & $1.87(0.86,4.07)$ \\
\hline \multirow[t]{2}{*}{ Resuscitated at birth } & Yes & $3.22(1.87,5.54)$ & $1.44(0.65,3.21)$ \\
\hline & No & 1 & 1 \\
\hline
\end{tabular}

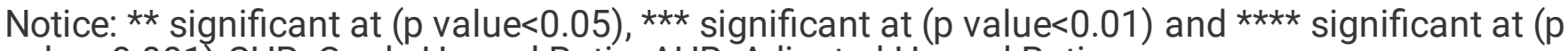
value $<0.001)$ CHR: Crude Hazard Ratio, AHR: Adjusted Hazard Ratio 


\begin{tabular}{|c|c|c|c|}
\hline Covariates & Category & $\mathrm{CHR}(95 \% \mathrm{Cl})$ & $\operatorname{AHR}(95 \% \mathrm{Cl})$ \\
\hline \multirow[t]{2}{*}{ EBF initiated within one hour } & Yes & 1 & 1 \\
\hline & No & $4.46(2.55,7.80)$ & $2.29(1.13,4.63)^{\star \star}$ \\
\hline \multirow[t]{2}{*}{ History of previous hospitalization } & Yes & $1.82(0.96,3.44)$ & $0.52(0.26,1.04)$ \\
\hline & No & 1 & 1 \\
\hline
\end{tabular}

\section{Discussion}

This study showed that the overall incidence rate of mortality was 20.5 per 1000 person days observation which was a little higher than the study conducted at Arba Minch, Southern, Ethiopia which was 14.57 per thousand person-days observation(1). This might be because of the difference in the follow up time that is, in our study we followed the neonates for the entire neonatal period but in the other study the neonates were followed for 14 days.

In this study, neonates with admission weight $<2500$ grams had 3.37 times higher risk of death compared to neonates with admission weight $>2500$ grams among neonates with neonatal sepsis and this was in line with a study done in India which had reported that neonates with admission weight $<2500$ grams to have an increased risk of mortality with sepsis (22). A similar study done in Indonesia had also reported that admission weight $<2500$ grams was significant predictor of mortality in neonates with neonatal sepsis (23). This might be due to low birth weight neonates over normal birth weight neonates have deficiencies in humeral and cellular immunity as well as high tendencies for prolonged hospitalization, which increases the risk of nosocomial infection.

In the current study, neonates who were delivered from mothers having history of intra partum fever had 7.3 times higher risk of mortality compared to neonates who were delivered from mothers who had no history of intra partum fever. This study is supported by a study done in Arba-minch, Ethiopia which had reported that neonates who were delivered from mothers having history of intra partum fever had 14 times higher risk of mortality compared to neonates who were delivered from mothers who had no history of intra partum. This study was also supported by a study conducted in Iraq which reported that neonates with neonatal sepsis who were born from mothers having history of fever were more likely to die than neonates with neonatal sepsis who were born from mothers who had no history of fever(24). Our study was also in line with a study conducted in India which reported that intra partum fever was significant predictor of mortality related to neonatal sepsis(25). This might be because the fact that the diseasecausing agent of maternal infection can ascend to the baby via circulation and during passage through the birth canal. 
Another predictor which was found significant in this study was place of delivery. Accordingly, consistent with a study result done at the university of Gondar comprehensive specialized hospital, neonates with neonatal sepsis who were delivered at home had 3.31 times higher risk of death compared to neonates who were delivered at health institution(26). The reason might be due to a lack of basic newborn care and environmental factors when the neonates were delivered at home.

Moreover in this study, the hazard of mortality among neonates with neonatal sepsis having late initiation of exclusive breastfeeding over an hour was 2.4 times higher compared to those neonates with neonatal sepsis who did get initiated to exclusive breast feeding within one hour. This finding is in line with to the studies conducted in India (27) and developing countries(28). This might be the fact that the many defense factors of the mother's milk include large amounts of secretory Immunoglobulin A antibodies produced by lymphocytes which have migrated from the mother's gut to the mammary glands. Thus breastfeeding modulates the early exposure of the neonate's intestinal mucosa to microbes and limits bacterial translocation through the gut mucosa and neonates who did not get initiated to exclusive breast feeding within one hour could not get this benefit from mother's milk.

The last predictor variable that predicted the neonatal mortality with sepsis was having comorbidities. In this regard our finding revealed that the hazard of mortality among neonates with neonatal sepsis having comorbidities was 1.8 times higher compared to those neonates without comorbidities. And this result is consistent with a study done in Iraq(24) which revealed that the presence of the likes of hypothermia would increase the likelihood of death among neonates with neonatal sepsis. This might be the fact that the presence of comorbidities would expose the neonate for further overwhelming of the immature immune.

\section{Conclusion}

In this study, high death incidence rate was observed. The mean time to death among neonates was 4.41 days. With regard to predictors: admission weight <2500 grams, comorbidity, late initiation of EBF over one hour, having history of intra partum fever and home delivery were found statistically significant predictors of mortality among neonates admitted with neonatal sepsis. We recommend future researchers to use longitudinal prospective cohort study design to avoid the drawbacks of retrospective cohort design and so as to not to miss important socio demographic predictors.

\section{Abbreviations}

AHR: Adjusted Hazard Ratio; ANC: Antenatal Care APGAR: Appearance, Pulse, Grimace, Activity and Respiration; APH: Anti Partum Hemorrhage; CHR: Crude Hazard Ratio; Cl: Confidence Interval; EBF: Exclusive Breast Feeding; EONS: Early Onset Neonatal Sepsis; ERB: Ethical Review Board; FHCSH: FelegeHiwot Comprehensive Specialized Hospital; ICU: Intensive Care Unit; IDR: Incidence Density Rate; LONS: Late Onset Neonatal Sepsis; NEC: Necrotizing Entrocolities; NICU: Neonatal Intensive Care Unit; PIH: Post Infectious Hydrocephalus; PROM: Premature Rupture of Membrane; PPROM: Prolonged Premature 
Rupture of Membrane; RDS: Respiratory Distress Syndrome; SSA: Sub Saharan Africa; TGSH: TibebeGhion Specialized Hospital; UNESCO: United Nations Educational, Scientific and Cultural Organization; UTI: Urinary Tract Infection; WHO: World Health Organization

\section{Declarations}

\section{Ethics approval and consent to participate}

Ethical clearance was obtained from Bahir Dar University College of medicine and health science Ethical Review Board (ERB). Then officials at different levels in the hospitals were communicated through letters. Confidentiality of the information was secured throughout the study process.

\section{Consent for publication}

Not applicable

\section{Availability of data and materials}

The datasets used and/or analyzed during the current study are available from the corresponding author on reasonable request

\section{Competing interests}

The authors declare that they have no competing interest

\section{Funding}

No funding organzation

\section{Authors' contributions}

"GT: prepared the manuscript, MT: prepared the proposal, analyzed, and interpreted the data and was a major contributor in writing of the manuscript. MB and AG: approve the proposal and participate in the data analysis and interpretation. MT, SM, HG and DZ reanalysis, reinterpreted and revised the manuscript substantially. All authors read and approved the final manuscript."

\section{Acknowledgements}

Our earnest gratitude goes to Institutional Review Board (IRB) Bahir Dar University, College of Medicine and Health sciences, for ethical review and approval. We also thank Bahir Dar University, College of medicine and health science for funding of this paper

We would like also to extend our acknowledgment to Felege Hiwot and Tibebe Ghion hospitals' staffs for their cooperation 
Our sincere gratitude and appreciation also goes to data collectors, supervisors and participants of the study.

\section{References}

1. Dessu S, Habte A, Melis T, Gebremedhin M. Survival Status and Predictors of Mortality among Newborns Admitted with Neonatal Sepsis at Public Hospitals in Ethiopia. International Journal of Pediatrics. 2020;2020:8327028.

2. Gebremedhin D, Berhe H, Gebrekirstos K. Risk Factors for Neonatal Sepsis in Public Hospitals of Mekelle City, North Ethiopia, 2015: Unmatched Case Control Study. PLOS ONE. 2016;11(5):e0154798.

3. Goldstein B, Giroir B, Randolph A, Sepsis MotICCoP. International pediatric sepsis consensus conference: Definitions for sepsis and organ dysfunction in pediatrics*. Pediatric Critical Care Medicine. 2005;6(1):2-8.

4. Huynh B-T, Kermorvant-Duchemin E, Herindrainy P, Padget M, Rakotoarimanana FMJ, Feno H, et al. Bacterial infections in neonates, Madagascar, 2012-2014. Emerging infectious diseases. 2018;24(4):710.

5. Ansari S, Nepal HP, Gautam R, Shrestha S, Neopane P, Chapagain ML. Neonatal septicemia in Nepal: early-onset versus late-onset. International journal of pediatrics. 2015;2015.

6. Chiabi A, Djoupomb M, Mah E, Nguefack S, Mbuagbaw L, Zafack J, et al. The clinical and bacteriogical spectrum of neonatal sepsis in a tertiary hospital in Yaounde, Cameroon. Iranian Journal of Pediatrics. 2011;21(4):441.

7. Organization WH. Every newborn: an action plan to end preventable deaths. 2014.

8. Ambey R, Gupta R. Committing to Child Survival: A Promise Renewed-Progress Report 2013. Australasian Medical Journal (Online). 2014;7(2):136.

9. Demographic CE. Health Survey-2011. Central Statistical Agency Addis Ababa. Ethiopia ICF International Calverton, Maryland, USA. 2012. 2016.

10. Shaha CK, Dey SK, Shabuj KH, Chisti J, Mannan M, Jashimuddin M, et al. Neonatal sepsis a review. Bangladesh Journal of Child Health. 2012;36(2):82-9.

11. Tiru B, DiNino EK, Orenstein A, Mailloux PT, Pesaturo A, Gupta A, et al. The Economic and Humanistic Burden of Severe Sepsis. PharmacoEconomics. 2015;33(9):925-37.

12. Ranjeva SL, Warf BC, Schiff SJ. Economic burden of neonatal sepsis in sub-Saharan Africa. BMJ global health. 2018;3(1).

13. Freitas F, Araujo A, Melo M, Romero G. Late-onset sepsis and mortality among neonates in a Brazilian Intensive Care Unit: a cohort study and survival analysis. Epidemiology \& Infection. 2019;147.

14. Kayange N, Kamugisha E, Mwizamholya DL, Jeremiah S, Mshana SE. Predictors of positive blood culture and deaths among neonates with suspected neonatal sepsis in a tertiary hospital, MwanzaTanzania. BMC pediatrics. 2010;10(1):39. 
15. Mugalu J, Nakakeeto M, Kiguli S, Kaddu-Mulindwa DH. Aetiology, risk factors and immediate outcome of bacteriologically confirmed neonatal septicaemia in Mulago hospital, Uganda. African health sciences. 2006;6(2):120-6.

16. Shobowale E, Ogunsola F, Oduyebo O, Ezeaka V. A study on the outcome of neonates with sepsis at the Lagos University Teaching Hospital. International Journal of Medicine and Biomedical Research. 2015;4(1):41-9.

17. Motara F, Ballot DE, Perovic O. Epidemiology of Neonatal Sepsis at Johannesburg Hospital. Southern African Journal of Epidemiology and Infection. 2005;20(3):90-3.

18. Bitew ZW, Alemu A, Ayele EG, Jember DA, Haile MT, Worku T. Incidence Density Rate of Neonatal Mortality and Predictors in Sub-Saharan Africa: A Systematic Review and Meta-Analysis. International journal of pediatrics. 2020;2020.

19. Alam MM, Saleem AF, Shaikh AS, Munir O, Qadir M. Neonatal sepsis following prolonged rupture of membranes in a tertiary care hospital in Karachi, Pakistan. The Journal of Infection in Developing Countries. 2014;8(01):067-73.

20. Camacho-Gonzalez A, Spearman PW, Stoll BJ. Neonatal infectious diseases: evaluation of neonatal sepsis. Pediatric Clinics of North America. 2013;60(2):367.

21. Setiawan C. Background Paper 6.23 Neonatal Conditions. Priority Medicines for Europe and the World A Public Health Approach to Innovation. 2013:1-50.

22. Meshram RM, Gajimwar VS, Bhongade SD. Predictors of mortality in outborns with neonatal sepsis: A prospective observational study. Nigerian Postgraduate Medical Journal. 2019;26(4):216.

23. Kardana IM. Incidence and factors associated with mortality of neonatal sepsis. Paediatrica Indonesiana. 2011;51(3):144-8.

24. Jumah DS. Predictors of mortality outcome in neonatal sepsis. The Medical Journal of Basrah University. 2007;25(1):11-8.

25. Bharad RV, Singh CS, Singh LR. Risk factors and immediate outcome of early onset neonatal sepsis. J Med Sci Res. 2017;5:21050-6.

26. Gebrehiwot A, Lakew W, Moges F, Moges B, Anagaw B, Unakal C, et al. Predictors of positive blood culture and death among neonates with suspected neonatal sepsis in Gondar University Hospital, Northwest Ethiopia. European Journal of Experimental Biology. 2012;2(6):2212-8.

27. Bandyopadhyay T, Kumar A, Saili A, Randhawa V. Distribution, antimicrobial resistance and predictors of mortality in neonatal sepsis. Journal of neonatal-perinatal medicine. 2018;11(2):14553.

28. Liang L, Kotadia N, English L, Kissoon N, Ansermino JM, Kabakyenga J, et al. Predictors of Mortality in Neonates and Infants Hospitalized With Sepsis or Serious Infections in Developing Countries: A Systematic Review. Frontiers in Pediatrics. 2018;6(277).

\section{Figures}




\section{Proportion of Neonatal Outcome}

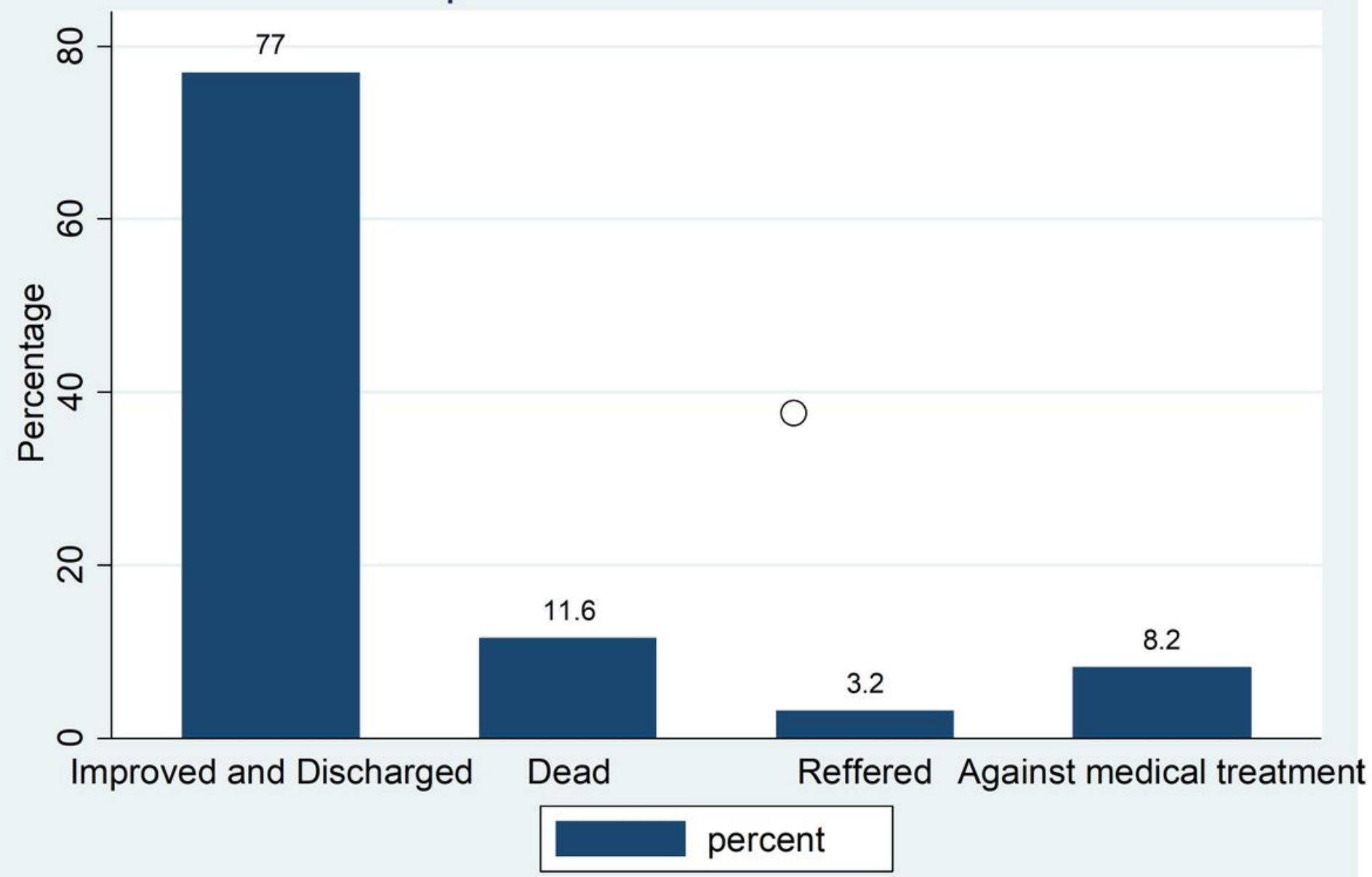

Figure 1

Proportion of neonatal outcome among neonates admitted with neonatal sepsis at public referral hospitals, Bahir Dar city, northwest, Ethiopia, 2021 ( $n=500)$ 


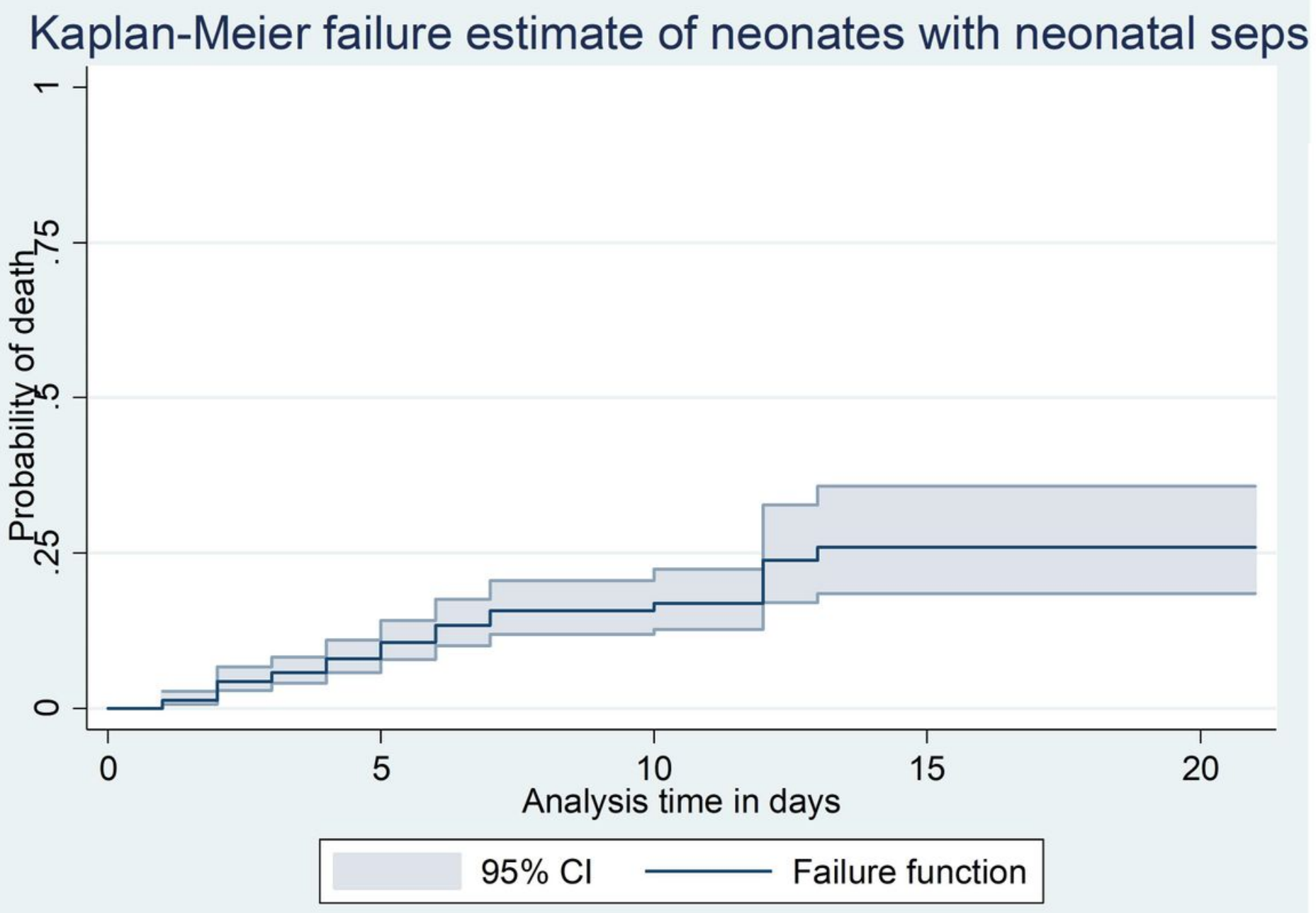

Figure 2

Overall Kaplan-Meir failure estimate of neonates admitted with neonatal sepsis at public referral hospitals, Bahir Dar city, northwest, Ethiopia, 2021 ( $n=500)$ 


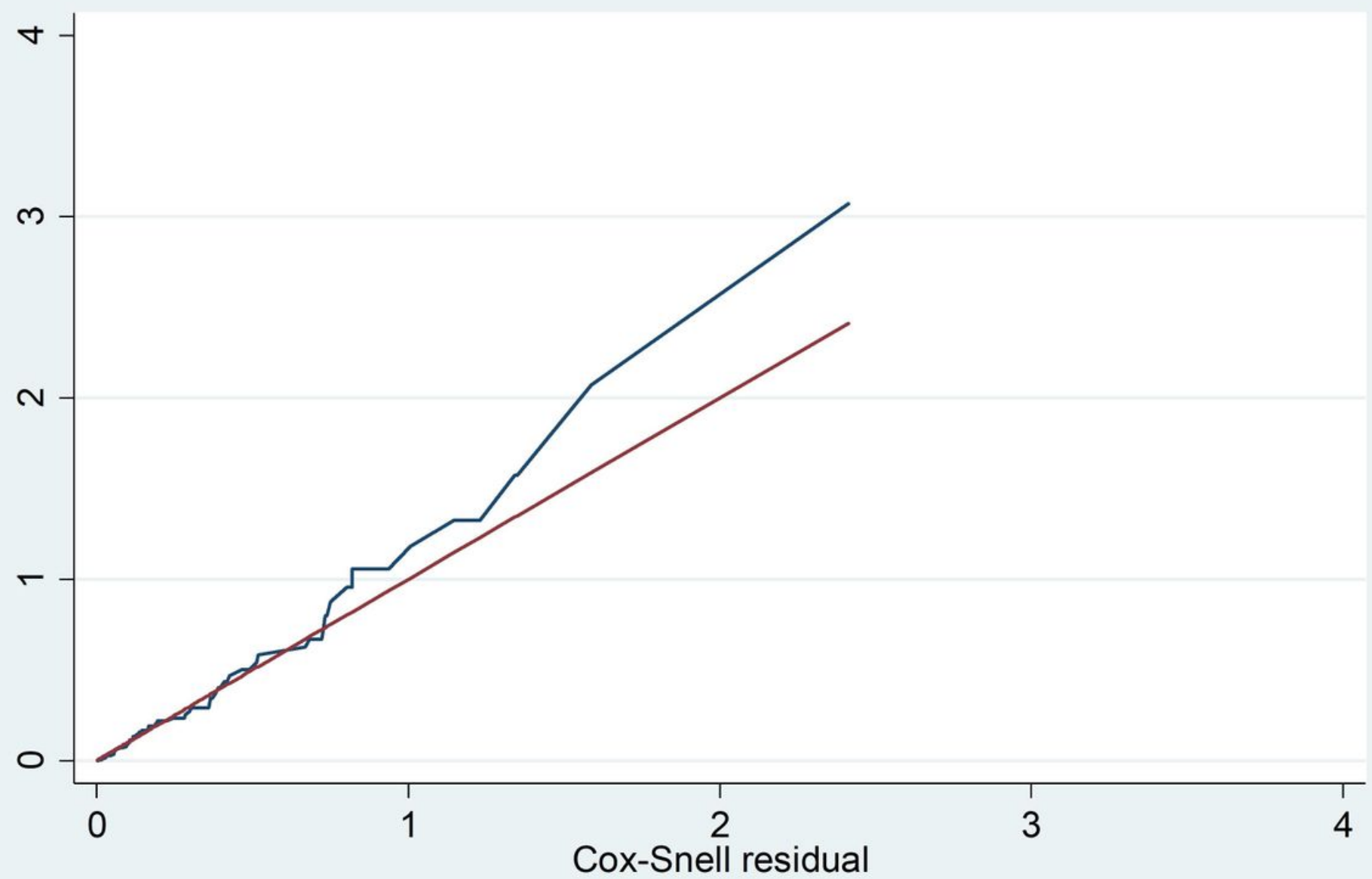

\section{Figure 3}

Cox-Snell residual Nelson-Aalen graph showing model goodness of fit for neonates admitted with neonatal sepsis at NICU of public referral hospitals Bahir Dar city, northwest, Ethiopia, $2021 \quad(n=500)$ 